\title{
Photofission-based, Nuclear Material Detection: Technology Demonstration
}

J. L. Jones, W. Y. Yoon, K. J. Haskell,

D. R. Norman, J. M. Hoggan Idaho National Engineering and Environmental Laboratory (INEEL)

C. E. Moss, C. A. Goulding, C. L. Hollas, and W. L. Myers

Los Alamos National Laboratory (LANL)

Ed Franco

Applied Research and Applications

Corporation (ARACOR)

December 2002

Idaho National Engineering and Environmental Laboratory Bechtel BWXT Idaho, LLC 



\title{
Photofission-based, Nuclear Material Detection: Technology Demonstration
}

\author{
J. L. Jones, W. Y. Yoon, K. J. Haskell, D. R. Norman, J. M. Hoggan \\ Idaho National Engineering and Environmental Laboratory (INEEL) \\ C. E. Moss, C. A. Goulding, C. L. Hollas, W. L. Myers \\ Los Alamos National Laboratory (LANL) \\ Ed Franco \\ Applied Research and Applications Corporation (ARACOR)
}

December 2002

Idaho National Engineering and Environmental Laboratory

Idaho Falls, Idaho 83415

Prepared for the

U.S. Department of Energy

Under DOE Idaho Operations Office

Contract DE-AC07-99ID13727 



\section{SUMMARY}

The Idaho National Engineering and Environmental Laboratory (INEEL), the Los Alamos National Laboratory (LANL), and the Advanced Research and Applications Corporation (ARACOR) [Sunnyvale, California] performed a photonuclear technology demonstration for shielded nuclear material detection during August 21-22, 2002, at the LANL TA-18 facility. The demonstration used the Pulsed Photonuclear Assessment Technique (PPAT) that focused on the application of a photofission-based, nuclear material detection method as a viable complement to the ARACOR Eagle inspection platform. The Eagle is a mobile and fully operational truck and cargo inspection system that uses a 6-MeV electron accelerator to perform real-time radiography. This imaging is performed using an approved "radiation-safe" or "cabinet safe" operation relative to the operators, inspectors, and any stowaways within the inspected vehicles. While the PPAT has been primarily developed for active interrogation, its neutron detection system also maintains a complete and effective passive detection capability.

Because a 6-MeV ARACOR Eagle system was not available for this technology demonstration, only a representative demonstration of the prototype detection system was possible. To simulate the nominal 6-MeV Eagle accelerator operation, a correlation method was developed that showed similar photonuclear production capability using a nominal $6-\mathrm{MeV}$ operation of the INEEL Varitron accelerator [1]. Since the Varitron does not have a "cabinet safe" operation mode as with the Eagle, this demonstration was performed within an approved LANL testing facility. In addition to providing the demonstration with the INEEL Varitron, the LANL facility allowed the use of a cargo container, special nuclear materials, and specific shield materials. Several INEEL and LANL neutron detectors were positioned around the cargo container. The placement of the neutron detectors in this demonstration was representative of the specific mounting locations (relative to the cargo container) on the Eagle's super-structure for a field application. Any expected variations in the detector locations used in this demonstration are not expected to significantly affect the overall results presented.

Based on the pre-selected delayed neutron acquisition time window (after each accelerator pulse), the demonstration showed the capability of detecting shielded nuclear materials within a cargo container using the Eagle's nominal operational condition despite encountering higher-than-expected neutron background. The higher background was primarily due to cosmic radiation effects at the high elevation ( 2134 meters) of the demonstration test site. The advantage of using higher-energy electron beam operations (up to $10 \mathrm{MeV}$ ) was also demonstrated. Nuclear material detection was shown to be easier and more pronounced at these higher energies. ARACOR has already started investigating possible Eagle modifications to enable these higher energy operations. While prompt photonuclear neutron emissions are typically several orders-of-magnitude larger, only delayed neutron emissions ( $\sim 5-20 \mathrm{~ms}$ after each accelerator pulse operating at $50 \mathrm{~Hz}$ ) are presented.

Both low-Z (atomic number) and high-Z shield materials for the nuclear material were used. High- $Z$ shielding (such as lead), while capable of providing 
effective attenuation of gamma rays from radioactive materials, had a limited effect in reducing photonuclear-induced neutron emissions generated from the copious number of highly penetrating, higher energy bremsstrahlung photons. Lower-Z materials (such as water and polyethylene) permit increased penetration of the interrogating photons, but are more effective in limiting the emissions of the induced neutrons.

Based on the current INEEL and LANL detector designs and their respective locations around the cargo container, the demonstration showed the detection of $4.8-\mathrm{kg}$ of highly enriched uranium and $4.4-\mathrm{kg}$ of depleted uranium surrounded by $1.27-\mathrm{cm}$ of lead or $20-\mathrm{cm}$ of water. While higher electron energy operations showed greater neutron emissions for a given configuration, the lowest energy operation (i.e., nominal $6.0-\mathrm{MeV}$ ) was limited by natural background-related counting uncertainties for this demonstration.

The fundamental feasibility of the integration of the ARACOR Eagle inspection system with the PPAT has been shown with this demonstration. The capability and detection sensitivity of the PPAT can be further improved with the use of higher electron beam energy operations and an enhanced neutron detection system design. Specifically, the detection system enhancements include: 1) minimizing the natural background contribution, 2) optimizing the data acquisition time window for delayed neutrons, 3 ) using the prompt neutron signal with the correlated Eagle image, and 4) maximizing the use of all induced emission signals (i.e., neutrons and gamma-rays). 


\section{CONTENTS}

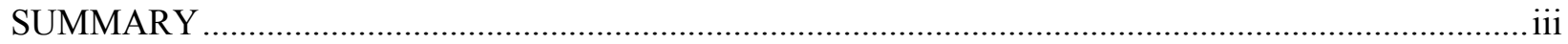

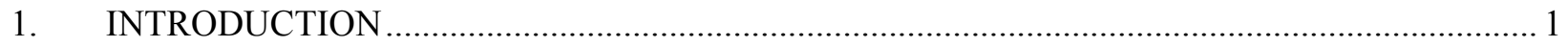

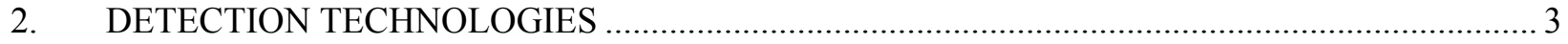

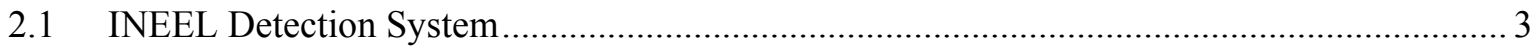

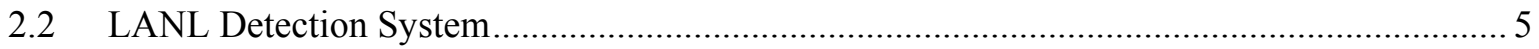

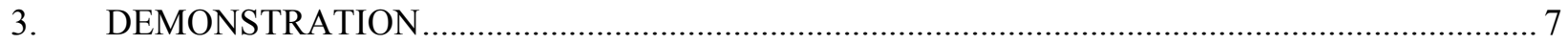

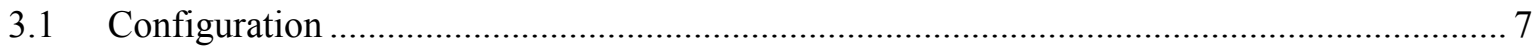

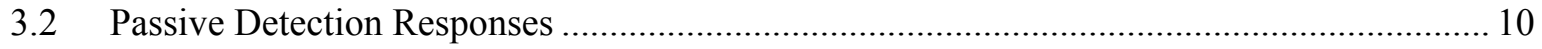

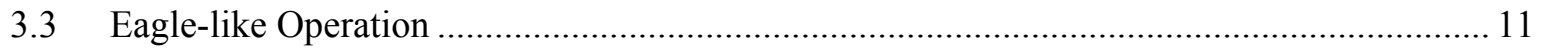

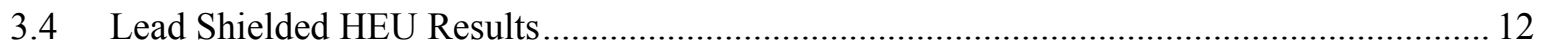

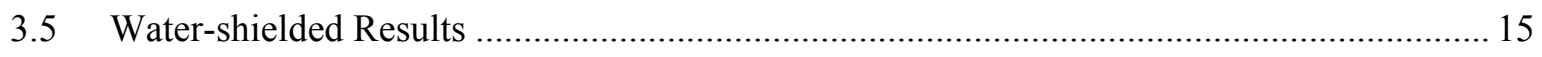

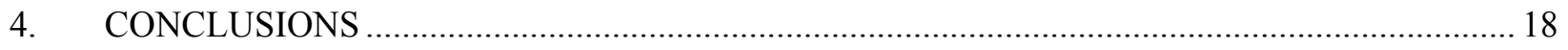

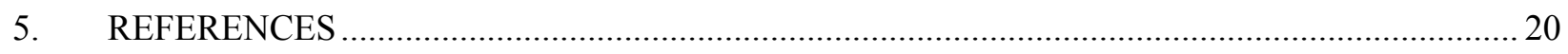

FIGURES

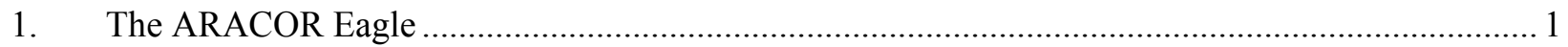

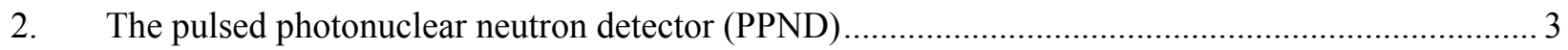

3. Calculated energy-dependent total neutron detection response for the PPND. (Source neutrons located at 100 centimeters from the PPND; "wall" represents a surrounding $61-\mathrm{cm}$-thick concrete structure at about 300 centimeters from the source.).................................... 4

4. The delayed neutron "region-of-interest" identified in two temporal detector responses:

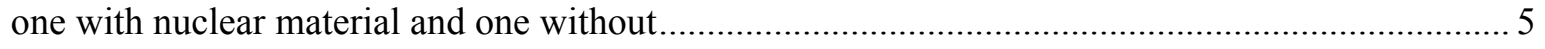

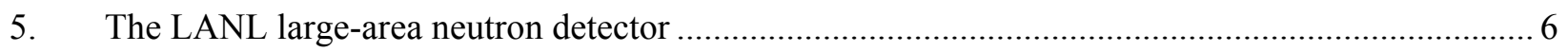

6. The transportable INEEL Varitron electron accelerator. The photon beam emerges to the right from the center of the circular shield on the aluminum table extension ........................... 7

7. Schematic showing the placements of the INEEL and LANL detectors relative to the cargo container and the electron accelerator's beam centerline and orientation 8 
8. Side detector group with and without borated-polyethylene back shielding ................................ 9

9. Top detector group with and without borated-polyethylene back shielding ................................ 9

10. The single INEEL detector positioned on top of the LANL detector.......................................... 9

11. $1.27-\mathrm{cm}$ thick lead shielding configuration used to shield nuclear material. Each lead shield side was approximately 30 -cm square

12. Varitron with forward uncollimated bremsstrahlung source. (Note the cylindrical tungsten shield housing and the exposed small electron/photon converter "button" at its radial center.)

13. Selected tungsten fan-beam collimator consisting of eight blocks. (Note, the addition of another tungsten disk structure [with a centered, 1-cm diameter hole] onto the shield housing of the electron/photon converter.)

14. Detector group responses using a $\mathrm{HEU} / \mathrm{Pb}$ target and a nominal 6-MeV accelerator operation with (w/) and without (w/o) the fan-beam photon collimator

15. Detector group responses using a $\mathrm{HEU} / \mathrm{Pb}$ target and a nominal $8-\mathrm{MeV}$ accelerator operation with $(\mathrm{w} /)$ and without (w/o) the fan-beam collimator

16. Detection assessment for various locations of the lead-shielded HEU within the container using a nominal 6-MeV accelerator operation with the fan-beam photon source

17. Detection assessment for various locations of the lead-shielded HEU within the container using a nominal 8-MeV accelerator operation with the fan-beam photon source.

18. Energy dependence of the detector groups for the shielded lead/HEU assembly positioned in the center of the container

19. The top view of the water-filled, 55-gallon drum. The circular inner container (shown with a handle) was used to provide a watertight seal for the nuclear materials

20. Detector group responses for a 4.8-kg HEU sphere positioned within a 55-gallon, water-filled drum located at the center of the cargo container.

21. Detector group responses for 4.8-kg HEU and 4.4-kg, rectangular DU plates positioned within a 55-gallon, water-filled drum located at the center of the cargo container using a nominal $8-\mathrm{MeV}$ accelerator operation

\section{TABLES}

1. Natural background responses for the INEEL and LANL detectors 


\section{Photofission-based, Nuclear Material Detection: Technology Demonstration}

\section{INTRODUCTION}

Detection of nuclear materials using a photofission-based, active interrogation technique (i.e., Pulsed Photonuclear Assessment Technique [PPAT]) was demonstrated at the Los Alamos National Laboratory (LANL) TA-18 facility during the week of August 19, 2002. The PPAT, using an electron accelerator with one or more specialized neutron detectors, has been developed for nuclear material detection for various applications $[1,2,3,4]$. The photonuclear investigative team of the Idaho National Engineering and Environmental Laboratory (INEEL), LANL, and Advanced Research and Applications Corporation (ARACOR) (Sunnyvale, California) conducted the test. The primary objective of this demonstration was to show the feasibility of complementing the effective radiographic imaging capability of the mobile ARACOR Eagle inspection system (see Figure 1) with a photofission-based, nuclear material detection capability. The Eagle is a nominal $6-\mathrm{MeV} \mathrm{x}$-ray inspection system that has successfully demonstrated "cabinet safe" operations and is currently deployed at the Port-of-Miami. The high energy operation of the Eagle allows the highest imaging performance with fully loaded cargo containers. Since nuclear materials have photofission threshold energies in the range of 5-6 MeV, the integration of the Eagle inspection system and the PPAT is being proposed using the nominal 6-MeV Eagle operation.

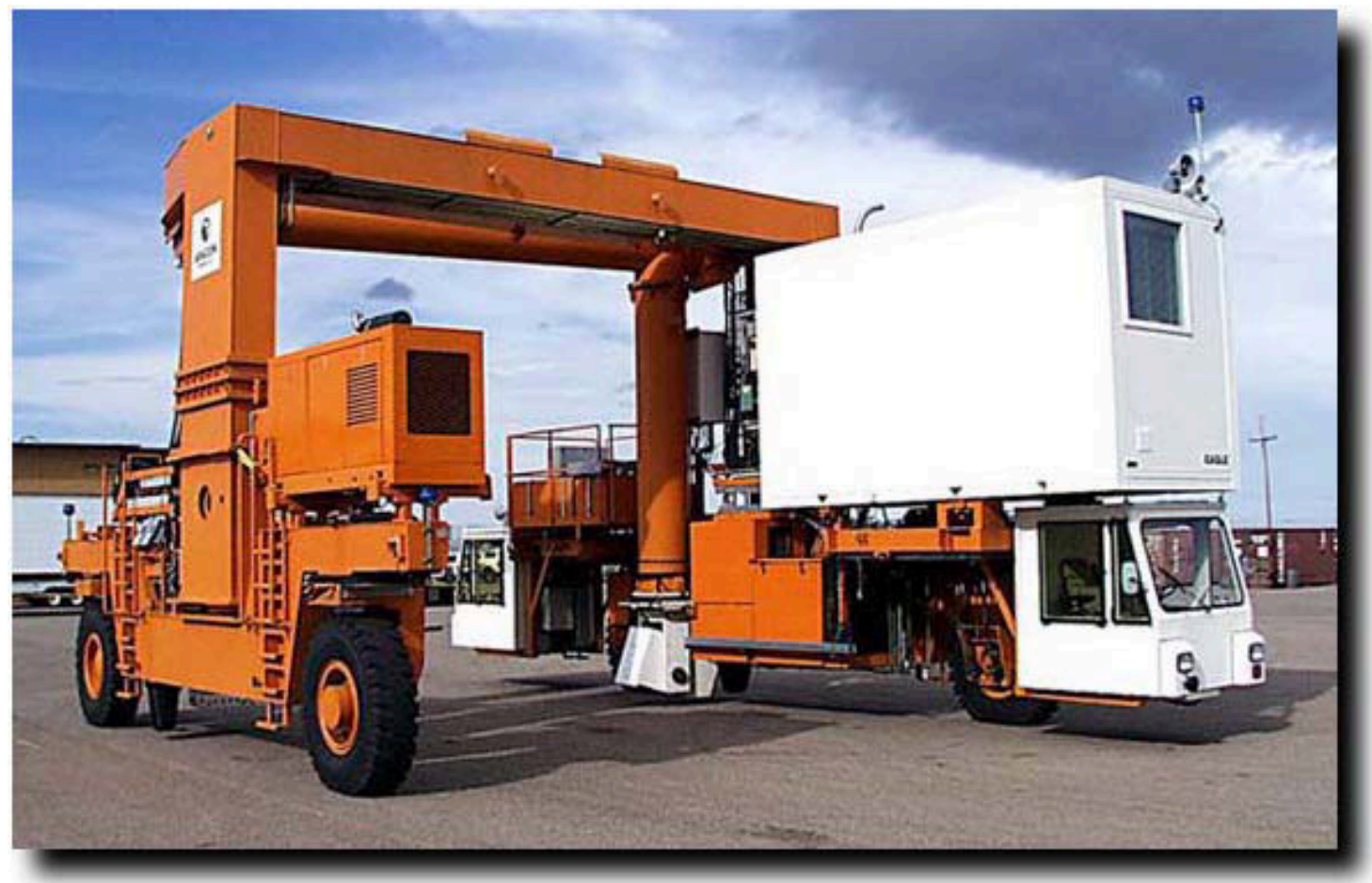

Figure 1. The ARACOR Eagle. 
The successful development of the PPAT detection technology would be extremely important to the nation's security. With the new technology integrated with the Eagle, routine x-ray inspections can be performed while simultaneously interrogating the cargo for the presence of any nuclear material. This combined approach would significantly increase the chance of nuclear material detection. Since (shielded) nuclear material is very dense, the inspector should easily be able to detect a suspicious area in the x-ray image. The current penetration depth for Eagle imaging is quoted at $300 \mathrm{~mm}$ for iron and about $2440 \mathrm{~mm}$ of water. While the image is being acquired, the nuclear material detection system will operate automatically and without operator intervention. If the active interrogation system provides a nuclear material alert or if an operator observes any suspicious item, the area-of-interest location can be correlated with a feature in the x-ray image. The Eagle can then perform a "dwell mode" operation. In "dwell mode" the Eagle is repositioned on the correlated location, or any other suspicious area, to provide increased confidence in the nuclear material detection. It is believed that the active detection technology can be integrated in such a way as to not impact the use of the Eagle for x-ray inspection and at a reasonable cost. In addition, the PPAT neutron detection system is also applicable to passive inspections.

Because a 6-MeV ARACOR Eagle system was not available for this demonstration at LANL, a correlation method was developed that showed similar photonuclear production capability using a nominal 6-MeV, $50 \mathrm{~Hz}, 3 \mu \mathrm{A}$ operation of the INEEL Varitron [4]. The nominal 6-MeV ARACOR Eagle operates with average beam currents much larger than $3 \mu \mathrm{A}$ with pulse repetition rates of up to 50 Hz. The difference in beam currents between these two machines reflect differences in their developed electron beam energy spectra below about $5-\mathrm{MeV}$. The nuclear materials used in this demonstration included plutonium-239, depleted uranium (DU), and highly enriched uranium (HEU). Highly enriched uranium is defined as uranium that has at least $80 \%$ enrichment in ${ }^{235} \mathrm{U}$. In addition to the use of a 244$\mathrm{cm} \times 244-\mathrm{cm} \times 305-\mathrm{cm}$ cargo container, the demonstration used lead and a 55-gallon drum of water as representative nuclear material shielding and investigated the use of electron beam energies up to 10 $\mathrm{MeV}$.

While all nuclear material is applicable to detection with the PPAT, the LANL demonstration highlighted HEU. HEU was demonstrated to be much more difficult to detect than plutonium due to its very low spontaneous neutron emissions and low-energy gamma-ray emissions. It was shown by passive gamma measurements that shielded HEU is very difficult to detect. However, PPAT allowed detection of even shielded nuclear material.

This report describes the INEEL and LANL detection systems used in this demonstration, describes the detector arrangement around a cargo container, and presents the experimental data. Both passive and active results are presented. Passive results show the importance of having active interrogation detection of shielded nuclear materials. The active results focus on lead and water shielding for HEU and DU. In addition to showing electron beam energy-dependent delayed neutron detection responses, responses for various locations of the shielded HEU within the container are also presented. 


\section{DETECTION TECHNOLOGIES}

Detection systems from INEEL and LANL were used in this technology demonstration. Both detection systems use ${ }^{3} \mathrm{He}$ detectors. For data consistency, all experimental results were derived from 120 -second total interrogation times. The INEEL detection responses are for a selected delayed neutron detection region-of-interest corresponding to 4.95-20 ms after each trigger pulse generated at $50 \mathrm{~Hz}$. The LANL detector system selected the delayed neutron region as 4-19 ms after each accelerator pulse. Because of the focus on fission delayed neutrons for this application, the detection of prompt neutrons have not been optimized, and hence, will not be presented. Yet, the prompt neutron emissions, occurring immediately after each accelerator pulse, are at least two orders of magnitude greater than delayed neutron emissions from any nuclear material. Shielding material will also considerably increase the prompt neutron response via photoneutron reactions depending on photoneutron energy thresholds and photon interaction probabilities. The real time monitoring of the prompt signal can significantly extend the detection sensitivities at all beam energy operations and will allow simultaneous cargo monitoring during the image-acquisition stage of an Eagle inspection. A prompt signal, with a correlated Eagle image, would be able to provide additional information regarding the cargo contents; maybe even help to notify the inspector of significant low-Z shielding material around a very dense object in the image.

\section{$2.1 \quad$ INEEL Detection System}

INEEL uses an IBM-compatible computer, associated commercial electronics, and the pulsed photonuclear neutron detector (PPND) shown in Figure 2. The PPND weighs approximately $16 \mathrm{~kg}$ and has been designed for the detection of $0.1-\mathrm{keV}$ to $1.0-\mathrm{MeV}$ neutrons (see Figure 3). The latter range is representative of many delayed fission neutron emissions.

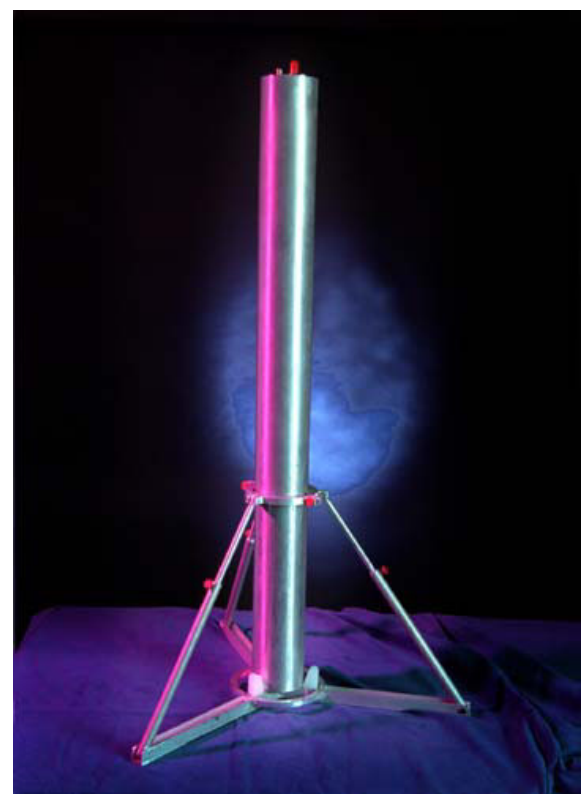

Figure 2. The pulsed photonuclear neutron detector (PPND). 


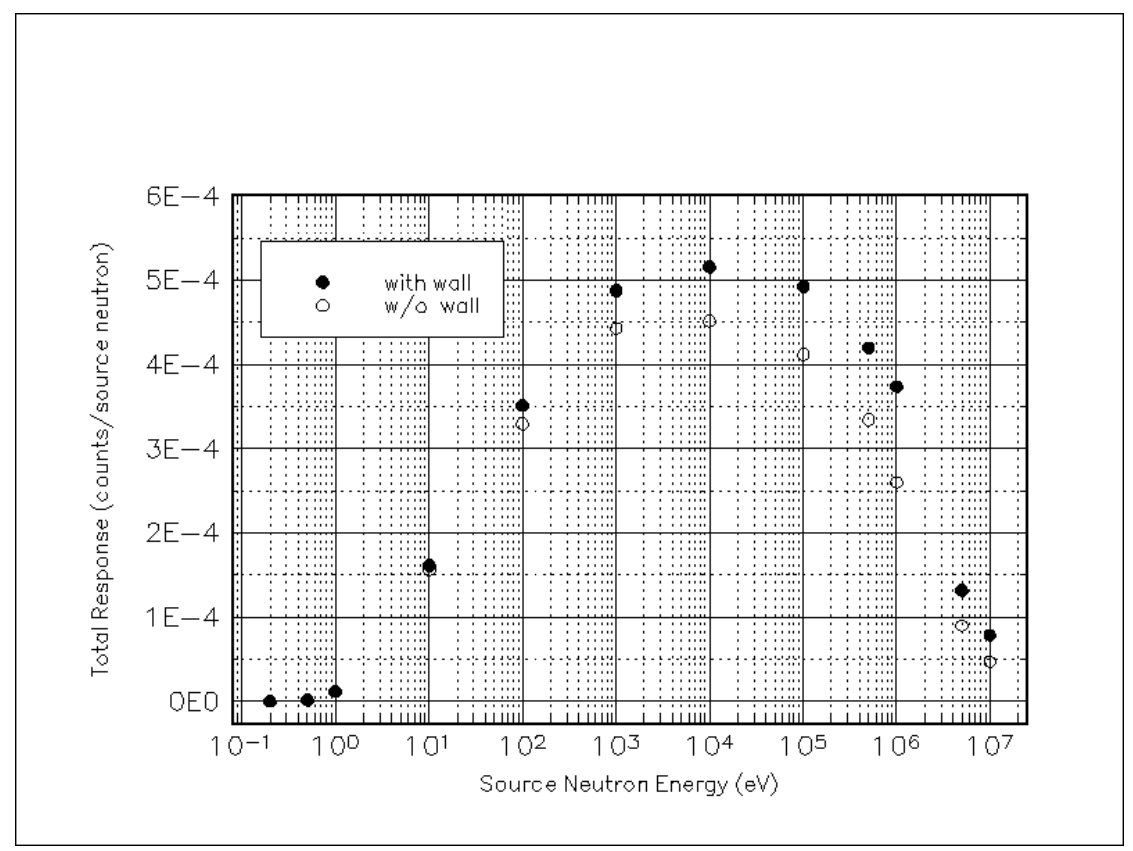

Figure 3. Calculated energy-dependent total neutron detection response for the PPND. (Source neutrons located at 100 centimeters from the PPND; "wall" represents a surrounding 61-cm-thick concrete structure at about 300 centimeters from the source.)

An INEEL-developed preamplifier (patent pending), located within the detector, has been specifically designed to operate within intense pulsed, photon-flash environments. The detector shielding design has been chosen to maintain a very low sensitivity to the voluminous room-return, low-energy neutrons associated with active interrogation environments. Figure 3 shows the PPND detector design effectiveness via the minimal detector response differences with and without a 61-cm-thick concrete wall at $\sim 300$ centimeters surrounding the detector. With this energy selectivity, the total passive detection efficiency of this detector is approximately $3 \times 10^{-4}$ counts per californium-252 $\left({ }^{252} \mathrm{Cf}\right)$ source fission neutron at 100 centimeters. With this detection efficiency, the PPND performs quite well and supports both passive and active detection applications. Specifically, the PPND is a 2.54-cm-diameter, 76.3-cmlong, 10 -atm, ${ }^{3} \mathrm{He}$ detector within a $117-\mathrm{cm}$-long, 10-cm-diameter aluminum housing also containing the high voltage power supply, the INEEL-built preamplifier, and concentric rings of polyethylene moderator, cadmium metal, and high-content, boron-loaded shielding. To assess electromagnetic noise sensitivities, the first PPND unit was housed in copper rather than aluminum. Each detector uses a small, internal $12-\mathrm{V}$ power supply to provide the high voltage to the ${ }^{3} \mathrm{He}$ tube. Twenty-three PPND detectors were assembled and deployed for this demonstration.

A multi-channel scaler electronic module, controlled by a personal computer-based data acquisition system (i.e., Genie-2000) and the accelerator's pulse trigger signal, allows the accumulation of all neutron counts after each accelerator pulse. The data is saved to the computer's hard drive. All data is acquired in 512 bins (note, the first bin records the number of accelerator pulses during the acquisition) with each bin being $39-\mu$ s wide for this application. By integrating the counts from selected bins, a user-selected region-of-interest count is identified. The delayed neutron region for this demonstration corresponded to channels 128 to 512. Figure 4 graphically identifies the delayed neutron "region-of-interest" for two temporal detector responses (i.e., counts vs. time). 

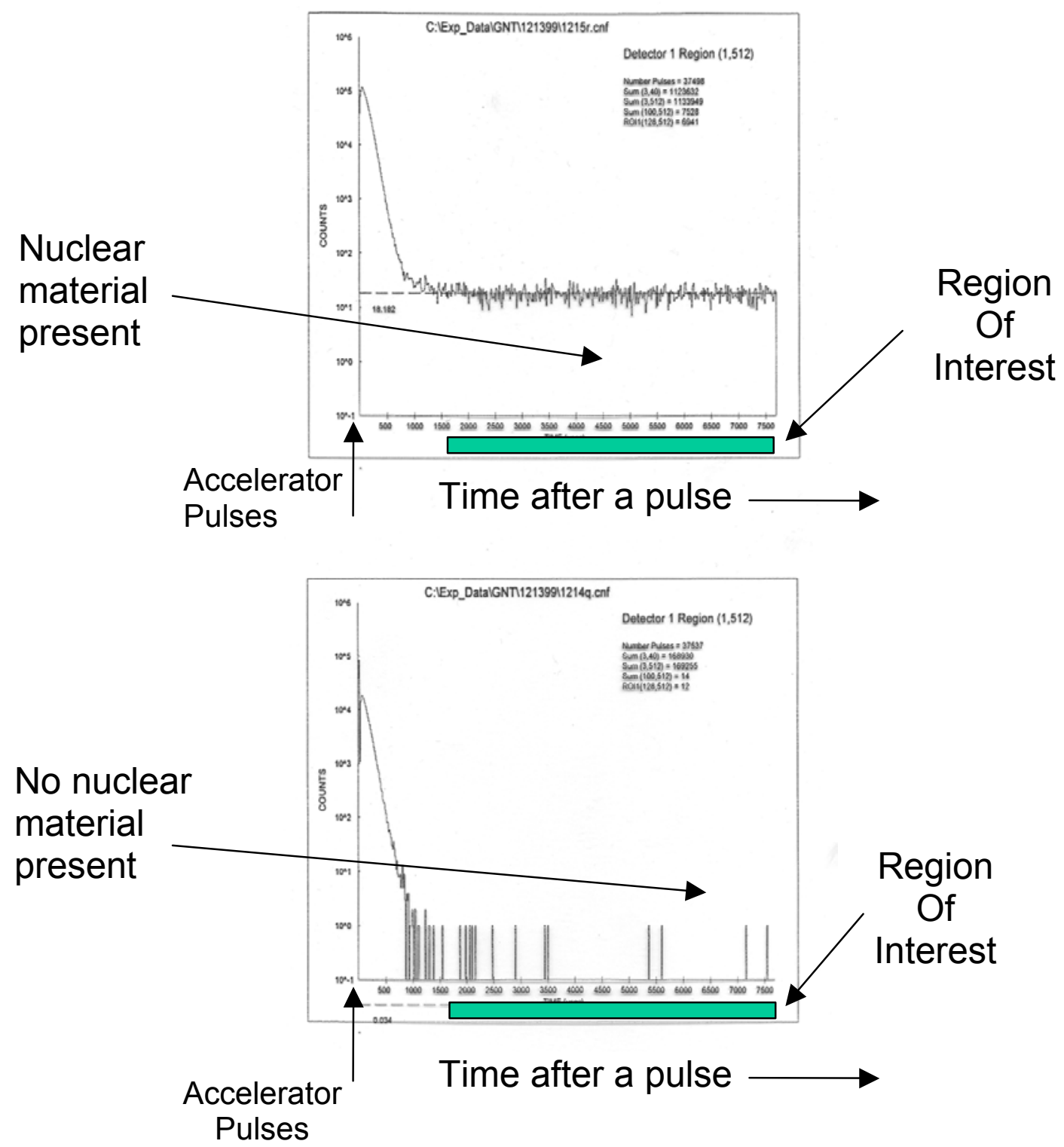

Figure 4. The delayed neutron "region-of-interest" identified in two temporal detector responses: one with nuclear material and one without.

\subsection{LANL Detection System}

The LANL detector (Figure 5) has a large area (122-cm high $\times 244-\mathrm{cm}$ long $\times 15-\mathrm{cm}$ thick) to maximize the solid angle for neutrons emitted by nuclear material. It contains four 5.0 -cm diameter $\times$ 182.88 -cm-long, 2 -atm, ${ }^{3} \mathrm{He}$ tubes arrayed within a high-density polyethylene box to moderate the neutrons. The polyethylene on the front (the large area facing the incident neutrons) is $1.27-\mathrm{cm}$ thick and on the back is 5.08 -cm thick. The detector is completely covered with 0.17 -cm-thick cadmium to prevent low-energy background neutrons from entering the detector. Because the original design of this detector attempted to maximize the efficiency-to-cost ratio, it contains a large amount of polyethylene, which is inexpensive, and very little ${ }^{3} \mathrm{He}$, which is expensive. The mass of $410 \mathrm{~kg}$ was not a concern in the original 
application as a vehicle monitor. The efficiency could be increased by using more ${ }^{3} \mathrm{He}$, but the increase is less than linear with the mass of ${ }^{3} \mathrm{He}$.

The electronics for processing the signals from the LANL neutron detector were a custom design developed for a larger array of detectors. The combined signal from the four ${ }^{3} \mathrm{He}$ tubes in each detector was fed into the preamplifier-amplifier discriminator emitter coupled logic module (PADEM) and pulse arrival-time recording module (PATRM) [5]. The PADEM produces a logic pulse for all signals above a threshold. The PATRM provides a time stamp for each event. The data were gated off for $\sim 2 \mathrm{~ms}$ during and after the beam pulse to allow the detector to recover from saturation and for all neutrons produced during the pulse to exit the detector. The data were stored event-by-event in the PATRM internal memory of one million 32-bit data words. At the end of each measurement the data were transferred to an IBM-compatible computer and saved on the hard drive.

The data were analyzed with the Feynman variance method [6,7] as implemented in a $\mathrm{C}++$ program. This program determines the number of correlated neutrons ("singles," "doubles," "triples," etc.) as a function of time after the beam pulse.

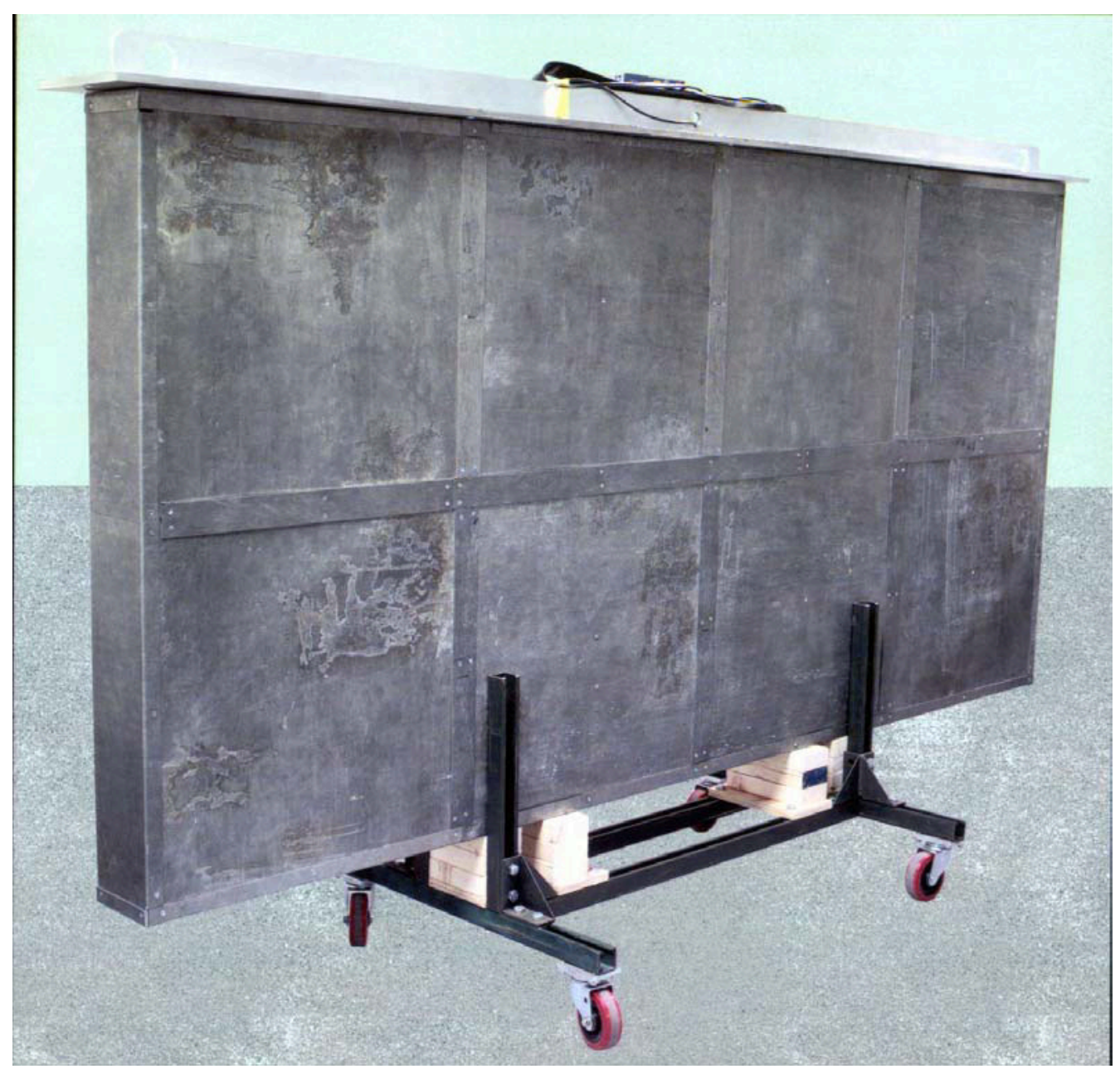

Figure 5. The LANL large-area neutron detector. 


\section{DEMONSTRATION}

The demonstration involved the use of a selectable-energy electron accelerator, the placement of neutron detectors around the inspected cargo container, and selected passive and active measurements as presented in this section.

\subsection{Configuration}

The interrogating photon beam was produced using the INEEL Varitron (a transportable electron accelerator) shown in Figure 6. To simulate the ARACOR-Eagle operational performance, the Varitron operated with a $50-\mathrm{Hz}$ pulse repetition rate, a peak electron beam energy of $6.1 \mathrm{MeV}$, and an average beam current of $\sim 3 \mu \mathrm{A}$. This matched performance is identified as the "nominal 6-MeV" operation. The Varitron is capable of producing higher user-selected, electron-beam energies of up to $12 \mathrm{MeV}$ and increased repetition rates to enable proportionally increased detection results.

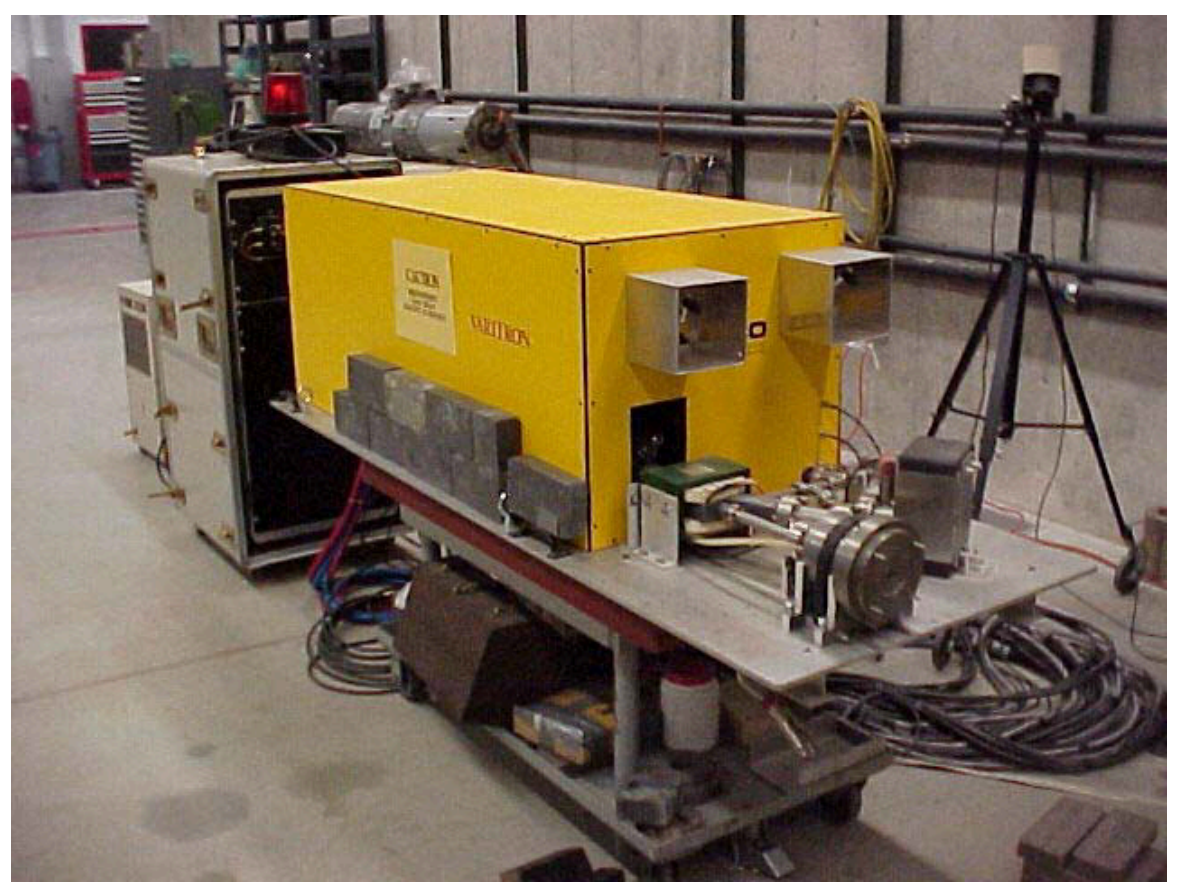

Figure 6. The transportable INEEL Varitron electron accelerator. The photon beam emerges to the right from the center of the circular shield on the aluminum table extension.

A $244-\mathrm{cm} \times 244-\mathrm{cm} \times 305-\mathrm{cm}$ cargo container was lowered into the basement of the LANL facility. The Varitron was positioned so that its bremsstrahlung source was $107 \mathrm{~cm}$ from one side of the container, and the accelerator's beam centerline was about $81 \mathrm{~cm}$ from the floor and perpendicular with the side of the container.

The LANL and INEEL detectors were positioned around the container as shown in Figure 7 . The figure also shows the accelerator's beam centerline orientation. The INEEL detectors are positioned in three groupings: twelve are along the side between the accelerator and the cargo container (two groups of six within $20-80-\mathrm{cm}$ of either side of the beam centerline) (see Figure 8), ten detectors equally spaced along the top of the container (see Figure 9), and a single, horizontally-positioned detector on top of the 
LANL detector on the direct opposite side of the container from the accelerator (see Figure 7 and 10). The latter INEEL detector was the original PPND assembled and was positioned to better enable response correlation between the INEEL and LANL detectors. The detector placements of the LANL and the INEEL side detector group were approximately $150 \mathrm{~cm}$ from the axial centerline of the cargo container. Because of the 244-cm height of the container and the typical placement of nuclear materials at about $60 \mathrm{~cm}$ from the container floor for this demonstration, the INEEL top group of detectors was positioned at least $184 \mathrm{~cm}$ from any nuclear material.

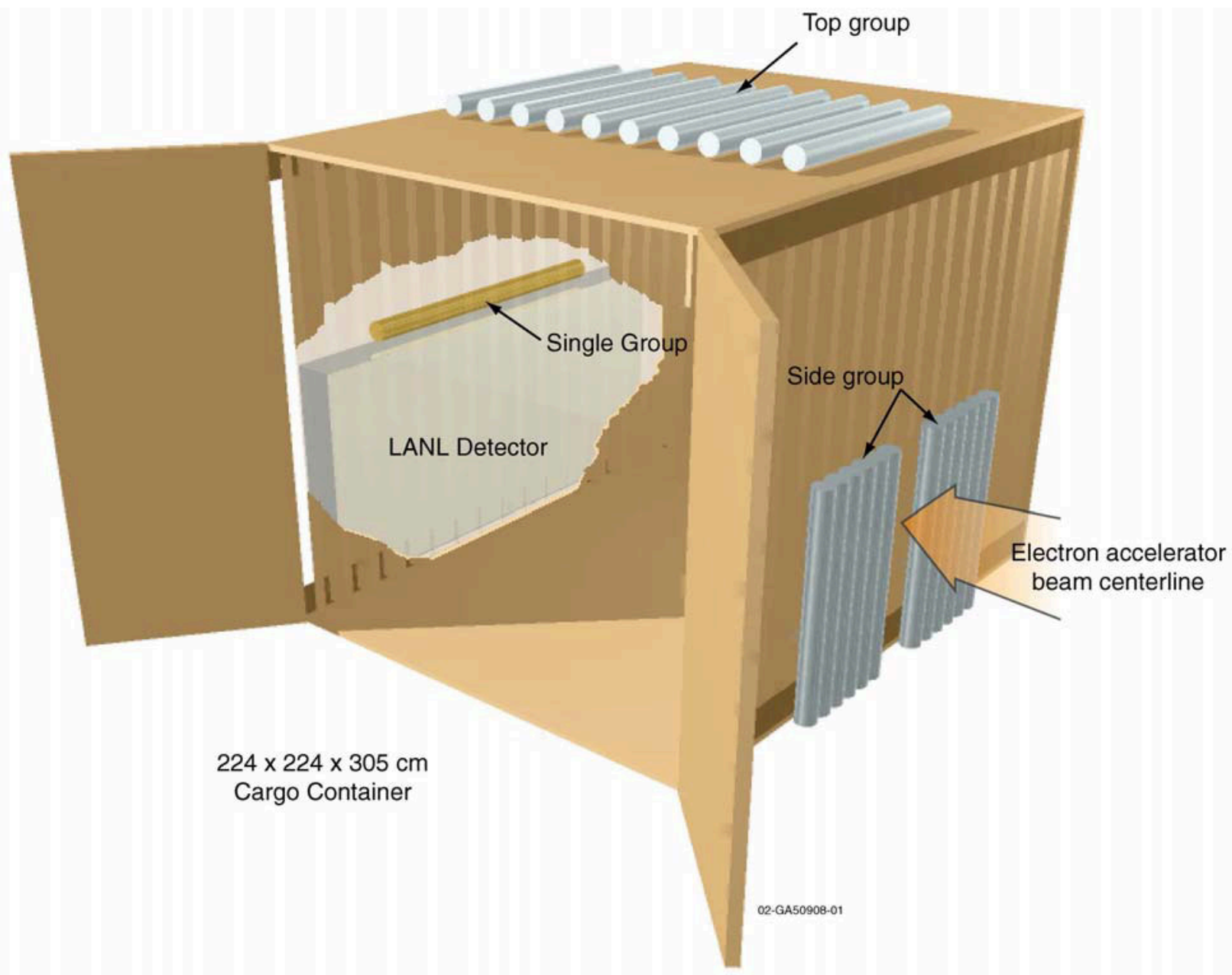

Figure 7. Schematic showing the placements of the INEEL and LANL detectors relative to the cargo container and the electron accelerator's beam centerline and orientation. 

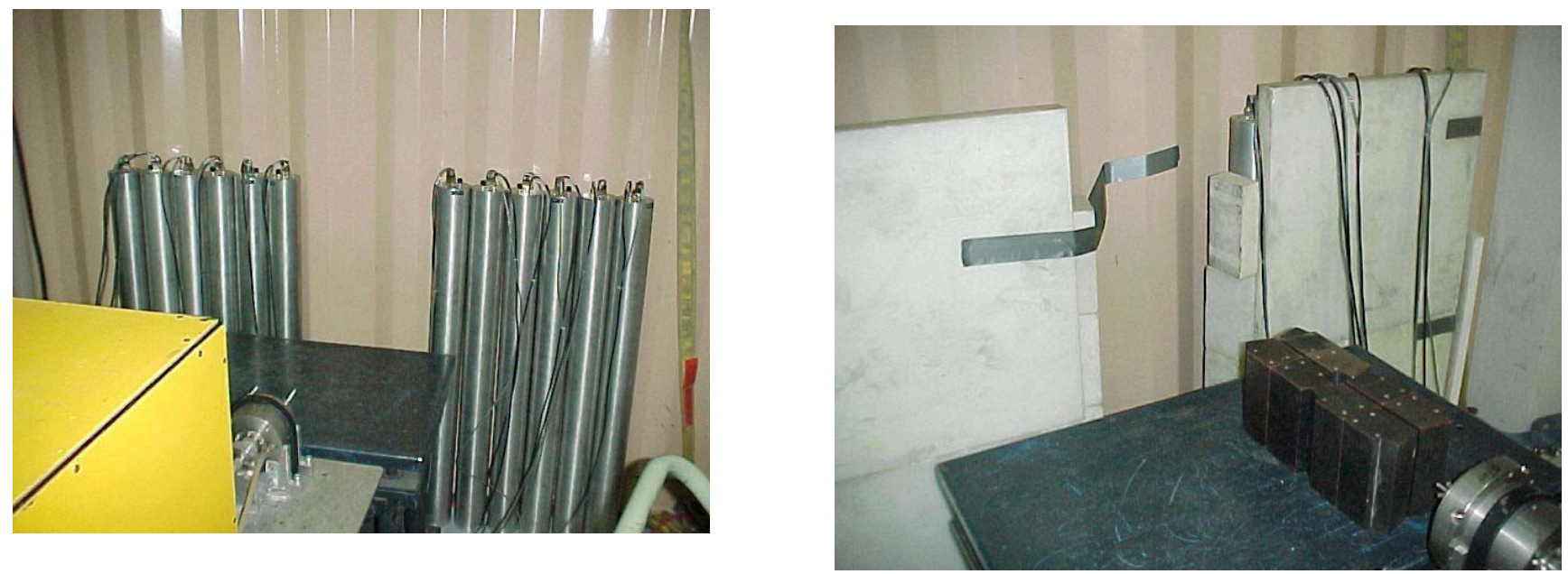

Figure 8 . Side detector group with and without borated-polyethylene back shielding.
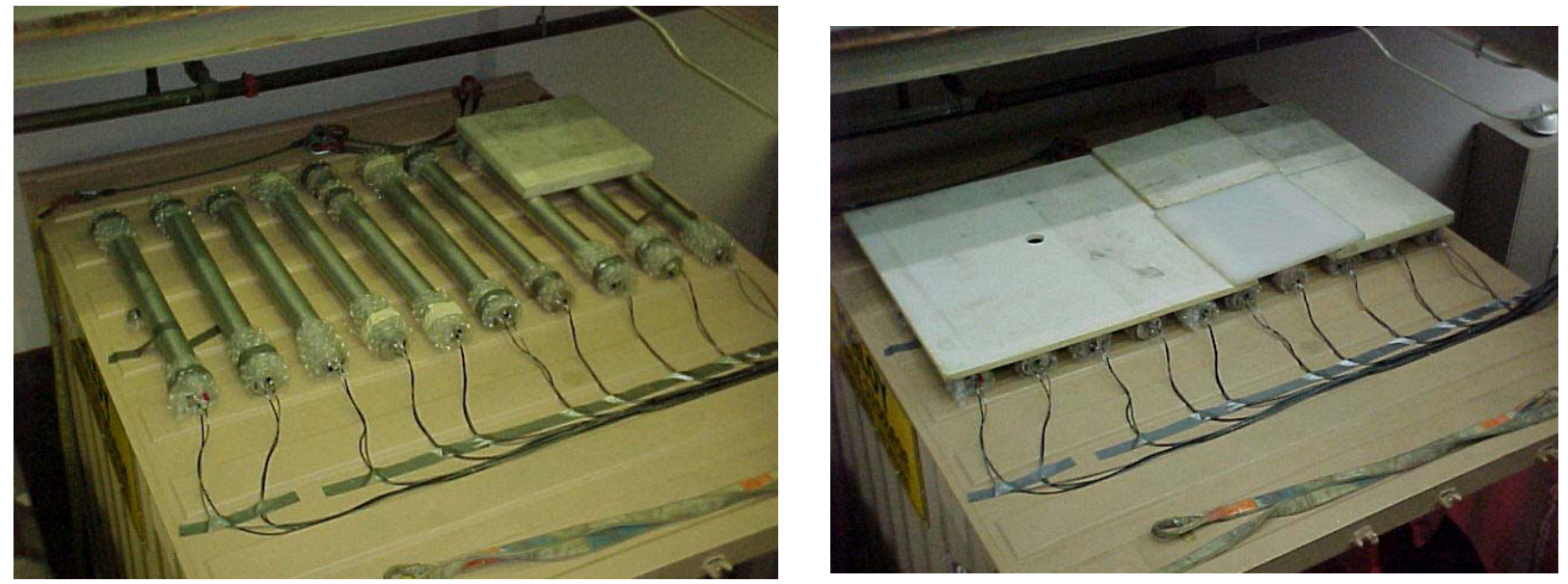

Figure 9. Top detector group with and without borated-polyethylene back shielding.

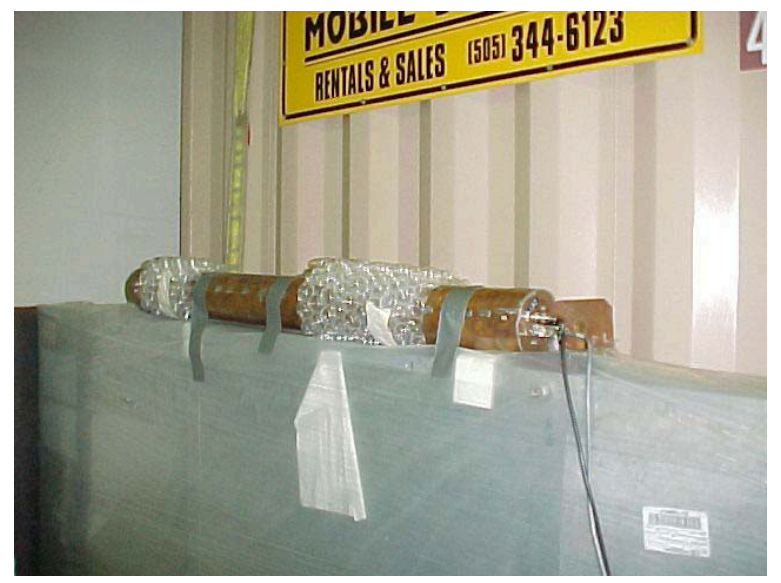

Figure 10. The single INEEL detector positioned on top of the LANL detector. 


\subsection{Passive Detection Responses}

The natural background of the LANL and the INEEL-grouped detectors are indicated in Table 1. The backgrounds for one INEEL detector can be compared with 0.11 counts per second measured near sea level [1], 0.03 counts per second measured in the Idaho Accelerator Center (IAC) (Pocatello, Idaho), and 0.5 counts per second in Idaho Falls, Idaho. Borated-polyethylene shielding was added as backing to the INEEL detectors (Figures 8 and 9) to provide approximately a 20\% reduction in the background responses. The "single" INEEL detector (see Figure 7), even though it was not directly shielded with borated polyethylene, did benefit indirectly from the shielding added to the other detectors, especially the top detector group. These background values were used to determine the net counts values presented in this paper.

Table 1. Natural background responses for the INEEL and LANL detectors

\begin{tabular}{|c|c|c|c|c|}
\hline \multirow[t]{2}{*}{$\begin{array}{c}\text { Additional Detector } \\
\text { Shielding }\end{array}$} & \multicolumn{3}{|c|}{$\begin{array}{l}\text { INEEL Detectors } \\
\text { (Counts/s) }\end{array}$} & $\begin{array}{l}\text { LANL Detector } \\
\text { (Counts/s) }\end{array}$ \\
\hline & Side & Top & Single & Unit \\
\hline None & $5.86 \pm 0.22$ & $6.03 \pm 0.22$ & $0.51 \pm 0.07$ & $2.51 \pm 0.15$ \\
\hline $\begin{array}{l}\text { With Borated } \\
\text { Polyethylene }\end{array}$ & $4.15 \pm 0.19$ & $4.83 \pm 0.20$ & $0.41 \pm 0.06$ & - \\
\hline
\end{tabular}

A radiation pager was used to show passive detection capabilities within a cargo container using 2 $\mathrm{kg}$ of plutonium-239 $(\mathrm{Pu})$ and $4.8 \mathrm{~kg}$ of HEU. The specific mass type and quantities were chosen based on representative special nuclear material availability and the requirements to stay within the safety limits for these tests at the test facility. The radiation pagers are small radiation sensors that alarm when they detect gamma rays and/or, for newer devices, neutrons from nuclear materials. In an unshielded nuclear material configuration and using the gamma-ray detection version of the radiation pager, the $\mathrm{Pu}$ was detected at distances much greater than the width of the cargo container, and HEU detection was increasingly more difficult. Using a 1.27-cm-thick lead shield (representative of a readily-available, minimally-thick, high density shield material) surrounding each material (see Figure 11), Pu was still detected with the pager but was sensitive to its location within the container. Shielded HEU detection was challenging.

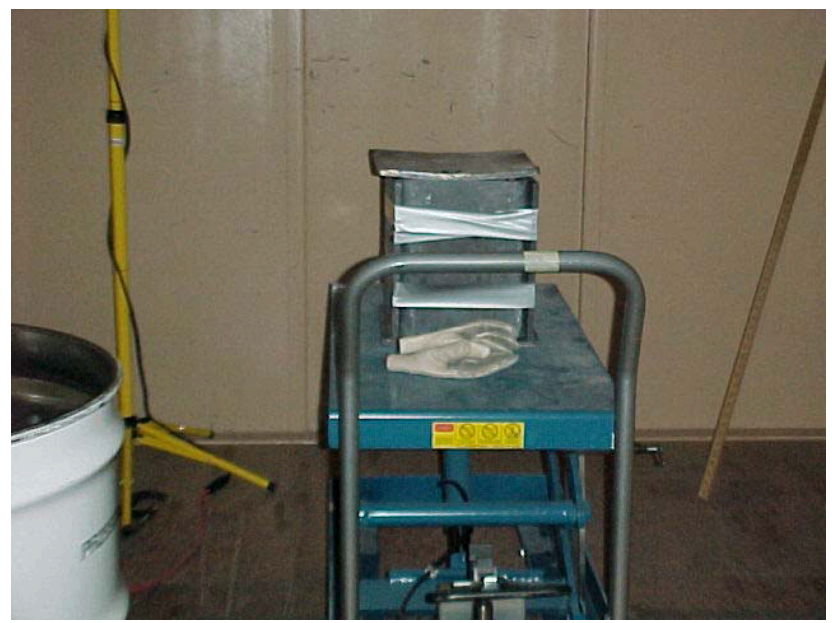

Figure 11. 1.27-cm thick lead shielding configuration used to shield nuclear material. Each lead shield side was approximately $30-\mathrm{cm}$ square. 
Before beginning any active interrogation demonstrations, the relative detection responses of both the INEEL and LANL detector systems were acquired simultaneously using a ${ }^{252} \mathrm{Cf}$ neutron source. The radioactive source was positioned at $100 \mathrm{~cm}$ from the nearest face of the long-axis of the LANL detector and $100 \mathrm{~cm}$ from the surface of one vertically oriented PPND. Specifically, a PPND was positioned within the container and $165 \mathrm{~cm}$ from the container wall nearest to the LANL detector. The front face of the LANL detector was outside the container and approximately $35 \mathrm{~cm}$ from the container wall. The source was vertically positioned on a stand so as to approximately match the half-height of both detectors. The larger LANL detector showed a net count rate of $106 \pm 1.3$ counts per second, while the PPND measured $5.02 \pm 0.2$ counts per second. Primarily due to the large size differences and LANL's multiple $\mathrm{He}^{3}$ tubes, the LANL detector $\left(\sim 30,000 \mathrm{~cm}^{2}\right)$ has approximately 21 times better passive detection efficiency than a single INEEL detector $\left(\sim 1,200 \mathrm{~cm}^{2}\right.$ projected area).

\subsection{Eagle-like Operation}

The ARACOR Eagle-like operation was determined for the INEEL Varitron (with a forward uncollimated bremsstrahlung photon source [see Figure 12]) at an April 2002 test in California using an Eagle-like accelerator [1]. A similar configuration was set up outside the cargo container using four INEEL detectors at $100 \mathrm{~cm}$ from a 4.4-kg DU target positioned at $105 \mathrm{~cm}$ forward of the photon source and along the accelerator's beam centerline. The net delayed neutron counting rates in the four detectors were $1.3 \pm 0.1,1.6 \pm 0.1,1.7 \pm 0.1$, and $1.5 \pm 0.1$ net counts per second. While these results are only slightly higher than the April measurements in California [1], the basic detection responses were considered acceptable for this demonstration.

Similar corresponding and acceptable results were obtained from the use of a photon fan-beam collimator (see Figure 13) to represent ARACOR's photon fan-beam imaging/interrogation capability. The selected tungsten collimation assembly does not attempt to simulate the proprietary design of the ARACOR Eagle collimator. The only purpose of the tungsten assembly was to assess the detection responses when less than the entire nuclear material configuration was illuminated with the high-energy photon beam. This approach allowed result extrapolations to the Eagle inspection system when providing continuous inspection from one end of a container to the other.

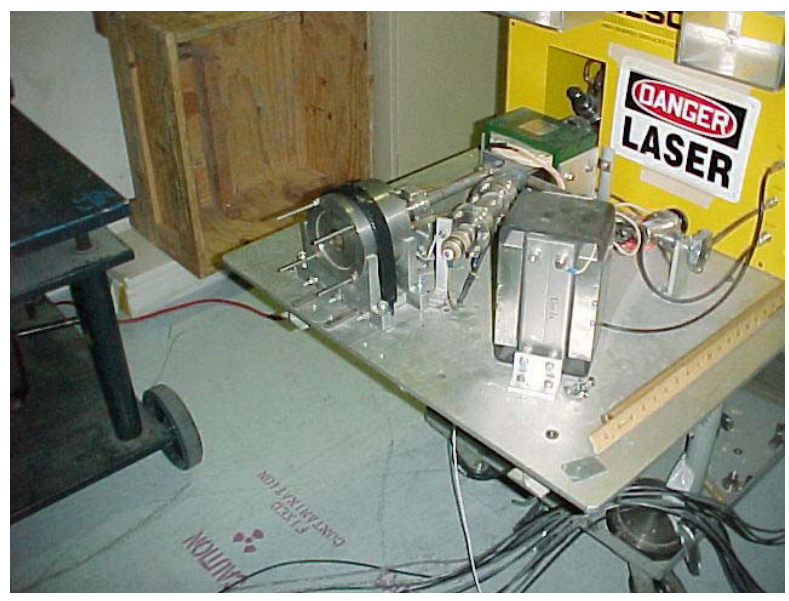

Figure 12. Varitron with forward uncollimated bremsstrahlung source. (Note the cylindrical tungsten shield housing and the exposed small electron/photon converter "button" at its radial center.) 


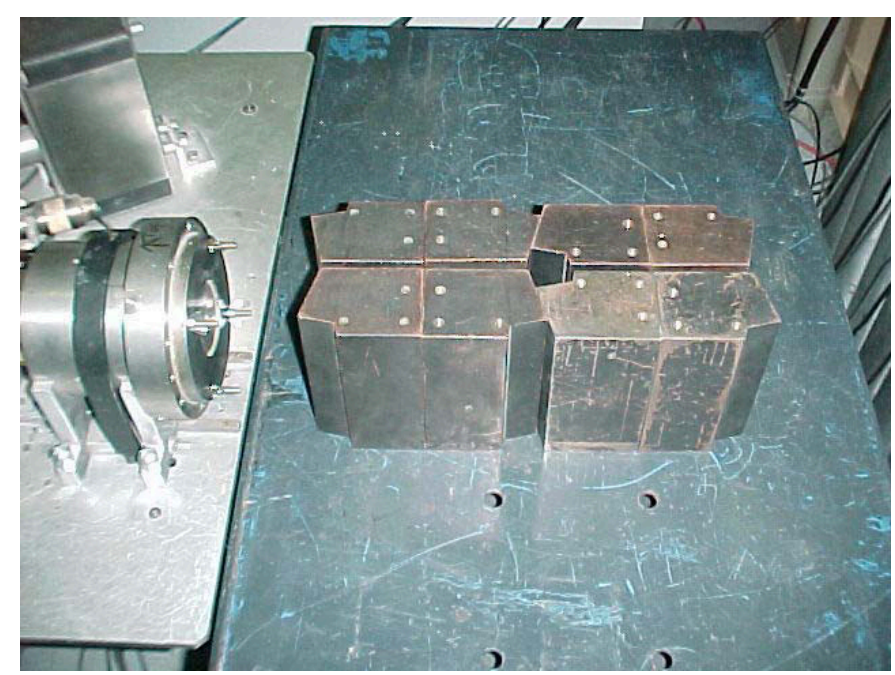

Figure 13. Selected tungsten fan-beam collimator consisting of eight blocks. (Note, the addition of another tungsten disk structure [with a centered, 1-cm diameter hole] onto the shield housing of the electron/photon converter.)

\subsection{Lead Shielded HEU Results}

The PPAT demonstration included a configuration with a 4.8-kg HEU sphere surrounded in a 1.27-cmthick lead shield (see Figure 11). The following results, with one standard deviation, were obtained with the uncollimated photon source (see Figure 12) and an ARACOR-representative fan-beam collimated configuration (see Figure 13) located between the photon source and the container. Figures 14 and 15 present delayed neutron detection responses from all the detector groups using a nominal 6-MeV and 8$\mathrm{MeV}$ electron beam operation, respectively. For example, from Figure 14, the LANL detector shows a net delayed neutron response of

$1.11 \times 10^{4}$ and $6.69 \times 10^{4}$ counts per beam coulomb, respectively, for the nominal 6-MeV operation corresponding to the "with" and "without" fan-beam collimation scenarios. Hence, using the $3 \mu \mathrm{A}$ beam current corresponding to the nominal 6-MeV operation, this results in a net detection rate of 0.20 and 0.33 counts per second, respectively. Similarly as shown in Figure 15, using the 4- $\mu$ A beam current corresponding to the nominal 8-MeV operation, the side detector group showed a net delayed neutron response of $4.81 \times 10^{6}$ counts per coulomb, or 19.2 net counts per second, for the "without fan" scenario. The rational for using specific nuclear material amounts and lead shielding was identified in Section 3.2. While a 4.8-kg sphere of HEU was used in this shielding demonstration, it must be noted that lower quantities of HEU can be detected with this interrogation technique and that other nuclear materials would provide similar photonuclear emission characteristics.

As can be observed, the nominal 6-MeV results are statistically limited due to lower photonuclear yields (or low total detection efficiencies) and, more importantly, higher-than-expected background contributions. Background suppression methods for a given detector system design will enable enhanced signal detection at this energy. As the electron beam energy is increased, the increased number of high energy photons will increase the photonuclear neutron emissions. Hence, the nominal 8-MeV operation results directly support nuclear material detection for this configuration. As expected, the selected fanbeam collimation does reduce the detection responses. Additionally, the INEEL side and top detector groups show larger 8-MeV operation detection responses than the LANL detector, while the single INEEL detector shows the expected "proportionally less" response. The larger response of the INEEL 
side and top detector groups at the $8 \mathrm{MeV}$ operation is probably due to the fact that these arrays contained much more ${ }^{3} \mathrm{He}$ than the LANL detector and the fact the INEEL detector/electronics have been specifically designed for operation in high photon flux environments. The fact that the responses of the LANL detector at the nominal $8 \mathrm{MeV}$ operation, with and without the fan-beam collimator, do not show more significant differences will require further investigation.

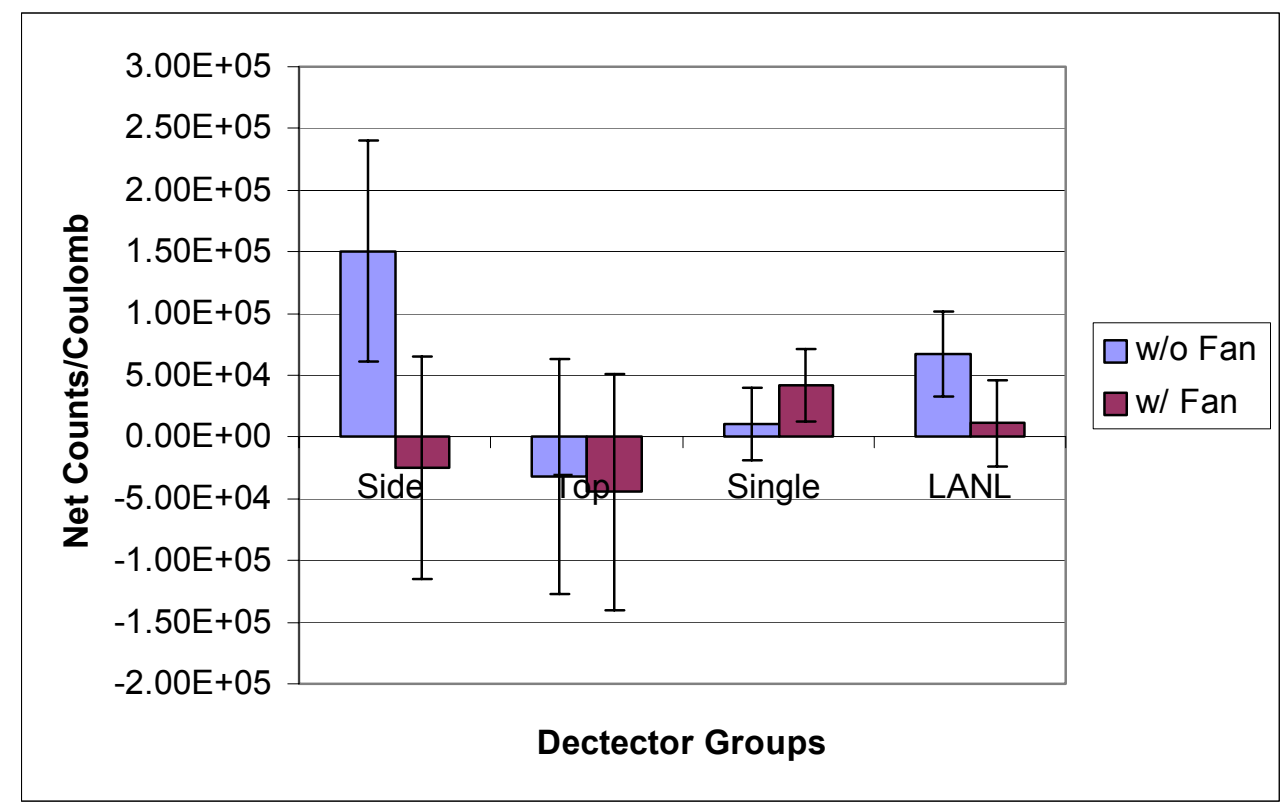

Figure 14. Detector group responses using a $\mathrm{HEU} / \mathrm{Pb}$ target and a nominal 6-MeV accelerator operation with (w/) and without (w/o) the fan-beam photon collimator.

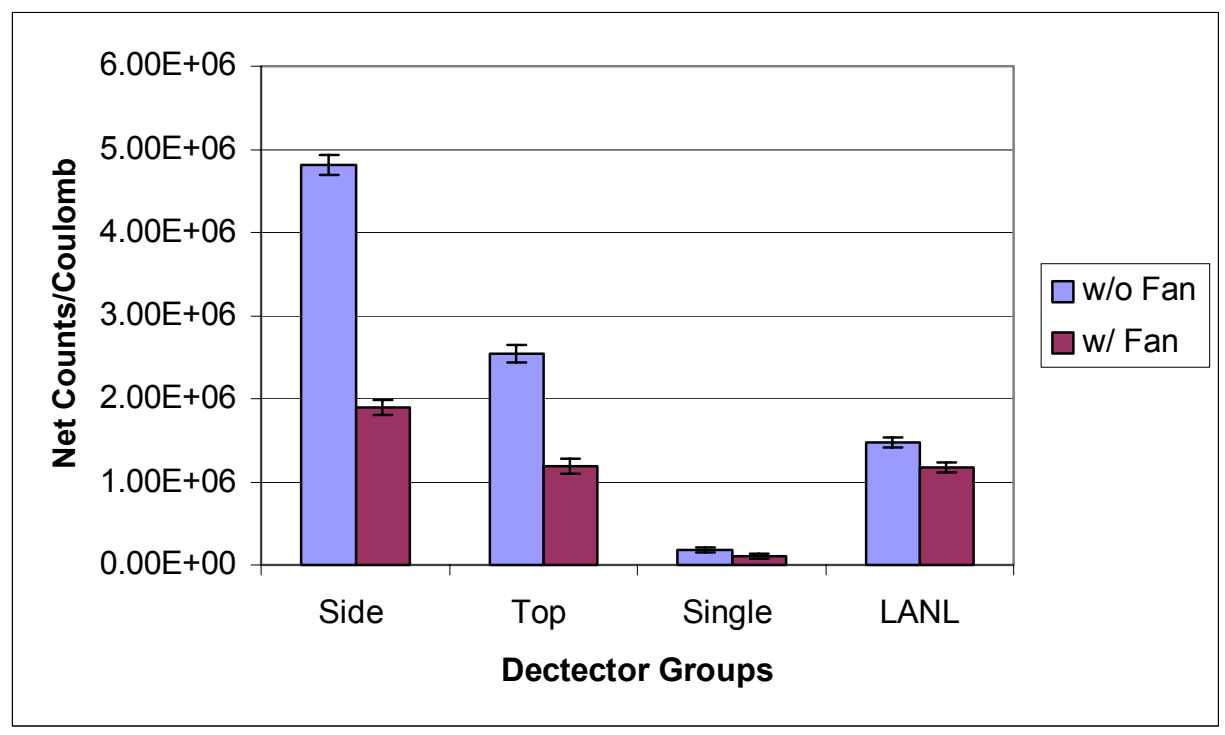

Figure 15. Detector group responses using a $\mathrm{HEU} / \mathrm{Pb}$ target and a nominal 8-MeV accelerator operation with (w/) and without (w/o) the fan-beam collimator. 
Using the selected photon fan-beam collimator, an assessment was made to observe the detection responses when varying the position of the shielded HEU along the accelerator beam centerline within the container. Figures 16 and 17 present the results of a nominal 6-MeV and $8-\mathrm{MeV}$ accelerator operation, respectively. The net count rates can be determined using the 3 and $4 \mu \mathrm{A}$ beam currents, respectively, for the nominal 6- and 8-MeV operations. As before, the statistical uncertainties limit the interpretation of the nominal $6-\mathrm{MeV}$ results. The nominal 8-MeV operation data suggest the need for detectors on both sides of a cargo container. The INEEL side detector group showed a decreasing detection response with increasing photon source-to-HEU distance; the LANL detector, due to its location opposite from the photon source, showed the corresponding increased detection response with decreasing detector-tonuclear material distance.

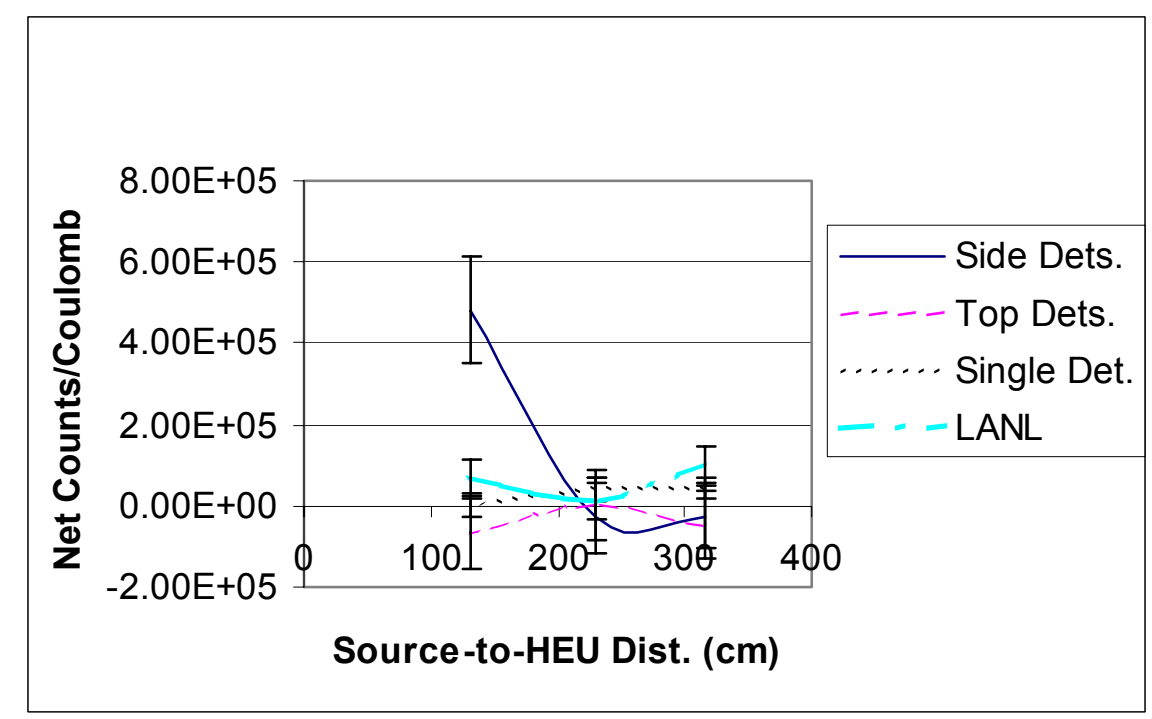

Figure 16. Detection assessment for various locations of the lead-shielded HEU within the container using a nominal 6-MeV accelerator operation with the fan-beam photon source.

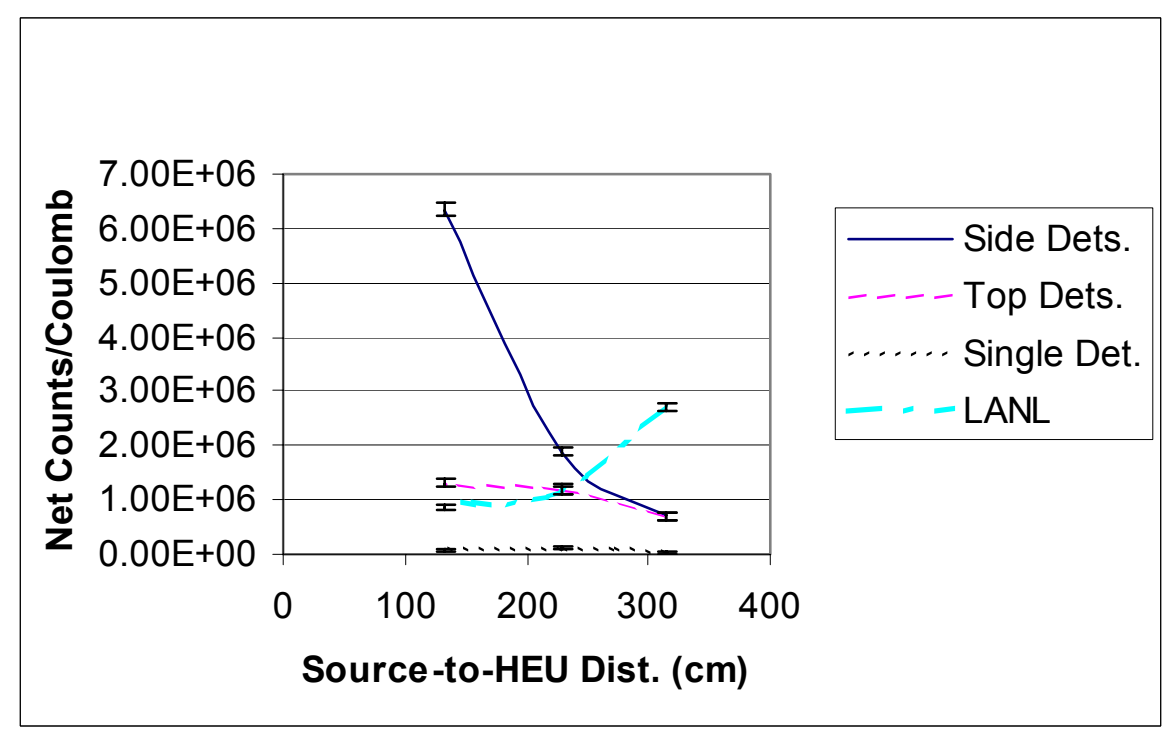

Figure 17. Detection assessment for various locations of the lead-shielded HEU within the container using a nominal 8-MeV accelerator operation with the fan-beam photon source. 
Using a forward uncollimated photon source and the lead shielded HEU located in the center of the cargo container, an assessment was made of the energy dependence of this technology. Figure 18 graphically displays the detection responses and the associated one sigma uncertainties. As indicated before, the net count rates can be readily determined using the average beam currents of 3,2.4, 4, and $1.4 \mu \mathrm{A}$ corresponding to the nominal 6-, 7-, 8-, and 10-MeV energy operations, respectively. The detection responses clearly increase with increasing electron-beam energy; the INEEL detectors show the fastest increase.

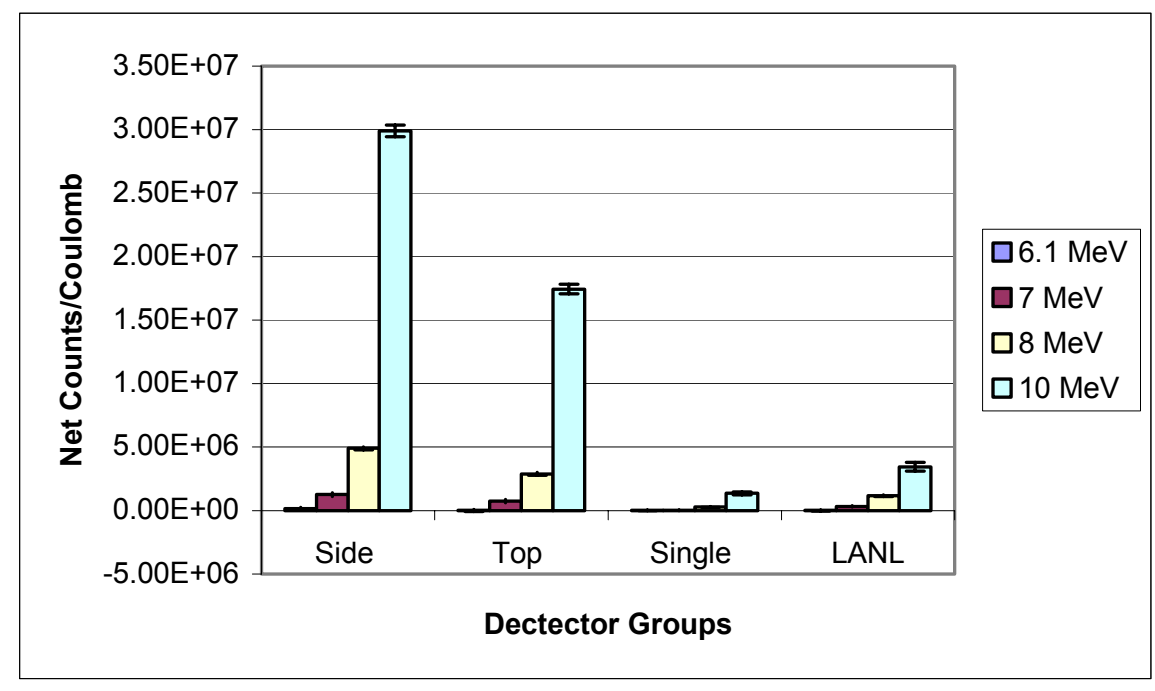

Figure 18. Energy dependence of the detector groups for the shielded lead/HEU assembly positioned in the center of the container.

\subsection{Water-shielded Results}

The final nuclear material shielding assessment involved the use of HEU and DU in a water-filled, 55-gallon drum (see Figure 19). Water was used since it is a representative low-density shield material with good neutron moderation properties and since liquids are common in commerce. It must be noted that this shielding configuration will be easily imaged with the Eagle and any suspect items would be applicable to these results.

The drum was filled with water and positioned at the horizontal center of the cargo container. No photon fan-beam collimation was used with the bremsstrahlung source in this shielding scenario. Based on post-test analysis, it has been determined that the HEU was positioned within the water at approximately $20-\mathrm{cm}$ below the accelerator's beam centerline. In addition, while at least $21 \mathrm{~cm}$ of water surrounded the HEU circumferentially and on the bottom, only $10-\mathrm{cm}$ of water was above the HEU due to the height of the watertight inner container. Figure 20 presents the results for this water-shielded HEU assembly for the nominal $8-\mathrm{MeV}$ and $10-\mathrm{MeV}$ accelerator operations. The side detector group showed a net detection response of $1.7 \mathrm{H} 10^{5}$ and $5 \mathrm{H} 10^{5}$ net counts per coulomb, or 0.68 and 0.70 counts per second, respectively, for the nominal 8 - and $10-\mathrm{MeV}$ operations.

Rectangular-shaped, 4.4-kg DU plates were also assessed within the water-filled drum. The DU plates were used since they had a mass similar to that of the HEU studied. In the latter case, only approximately $1 \mathrm{~cm}$ of water covered the top due to the need of a taller, watertight inner can. Figure 21 presents these results for the nominal $8-\mathrm{MeV}$ operation. The reduced water shielding, as seen by the top detector group with a 5.8 net counts per second response (i.e., $1.45 \mathrm{H} 10^{6}$ counts/ $\mathrm{C} * 4 \mu \mathrm{A}$ ), is clearly evident when DU was used. 


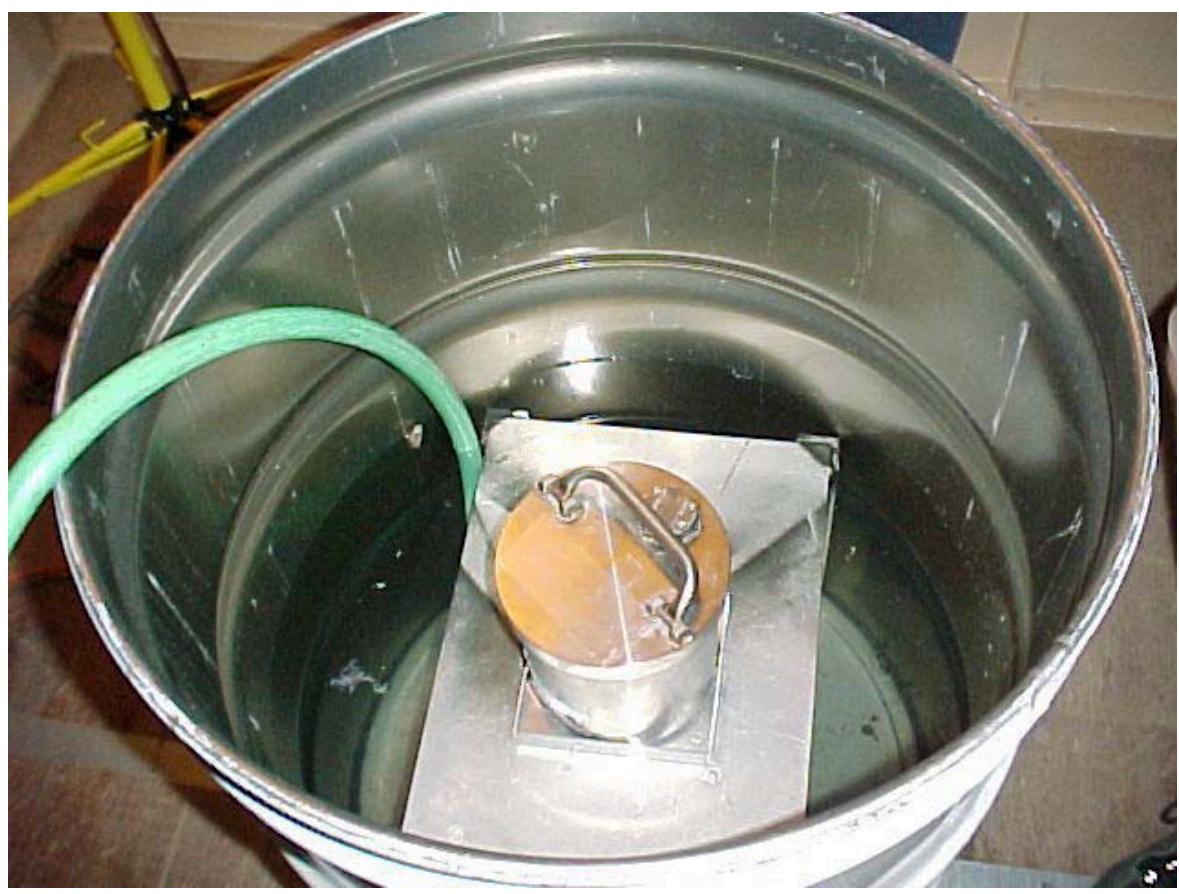

Figure 19. The top view of the water-filled, 55-gallon drum. The circular inner container (shown with a handle) was used to provide a watertight seal for the nuclear materials.

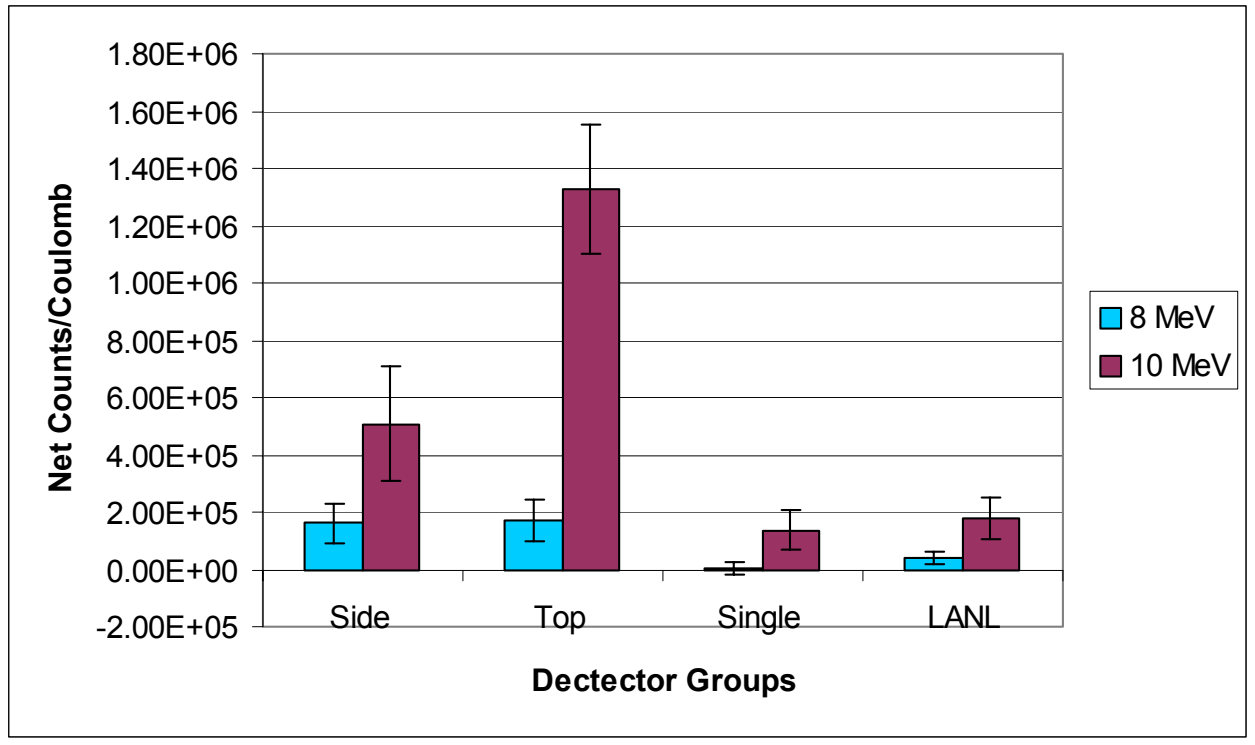

Figure 20. Detector group responses for a 4.8-kg HEU sphere positioned within a 55-gallon, water-filled drum located at the center of the cargo container. 


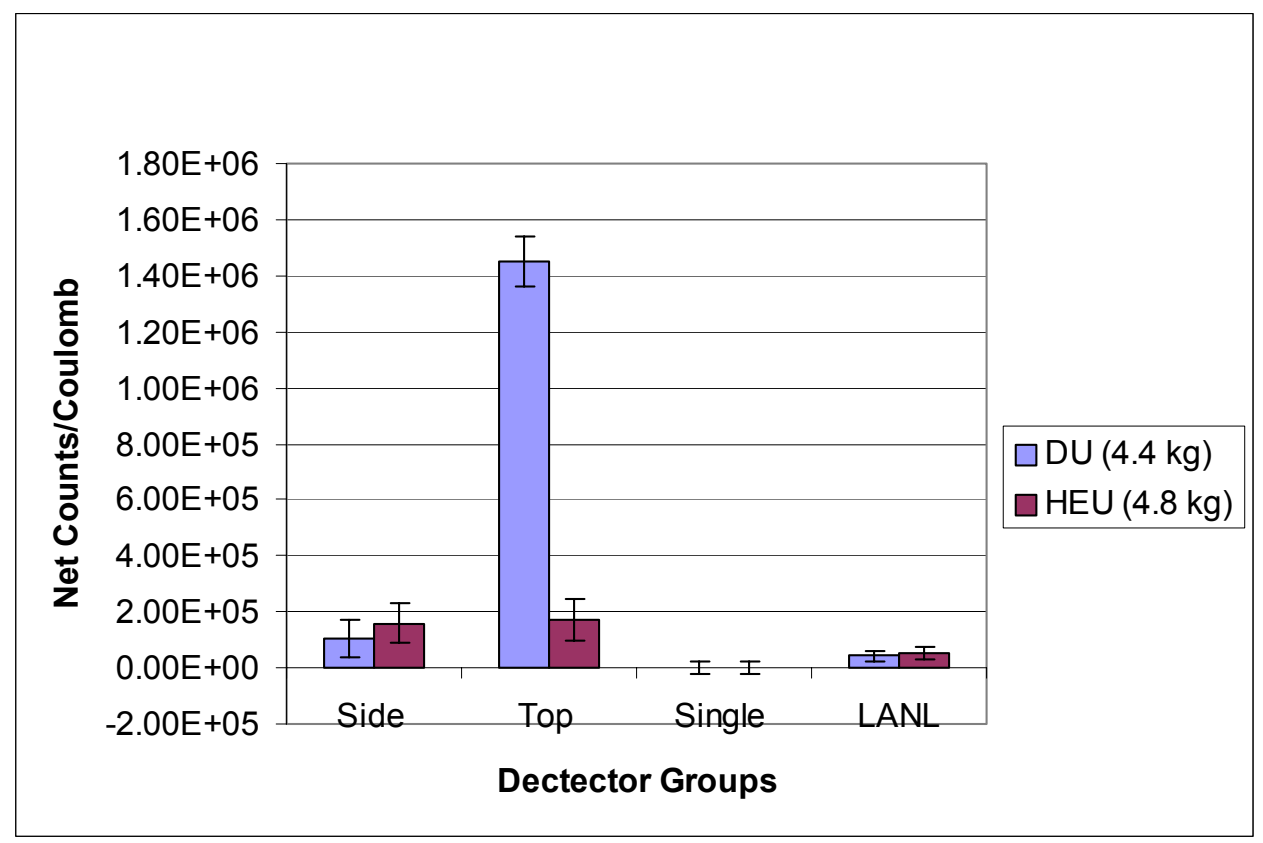

Figure 21. Detector group responses for 4.8-kg HEU and 4.4-kg, rectangular DU plates positioned within a 55-gallon, water-filled drum located at the center of the cargo container using a nominal $8-\mathrm{MeV}$ accelerator operation. 


\section{CONCLUSIONS}

The Pulsed Photonuclear Assessment Technique (PPAT), focusing on a photofission-based, detection technology, was demonstrated at the LANL TA-18 facility to assess its potential integration with the Eagle inspection system manufactured by ARACOR. Two modes of operation are being considered for the enhanced Eagle system. The first mode is to monitor the induced neutron emissions during the image acquisition process. The second mode involves a "dwell mode" operation. In the latter mode, the ARACOR Eagle, after having identified a suspect item from the real-time image, is repositioned at the suspect location and an interrogation is performed at that location to determine if nuclear material is present.

The LANL demonstration reproduced conditions similar to those encountered during an ARACOR Eagle inspection. The experimental conditions approximated the following conditions:

- $\quad$ Representative distances and locations for the deployment of the INEEL Varitron accelerator and neutron detectors relative to a cargo container.

- $\quad$ The x-ray beam was collimated to approximate the fan beam used by the Eagle for imaging.

- $\quad$ The operating conditions of the Varitron accelerator were matched to those of the Eagle accelerator that has a nominal 6-MeV operation with repetition rate of about $50 \mathrm{~Hz}$.

- $\quad$ Representative nuclear material quantities were shielded and placed inside the cargo container in various locations.

The demonstration used depleted uranium and weapons-grade materials to highlight some important passive detection issues and concerns related to shielded nuclear material detection within a cargo container. The active interrogation demonstration showed that lead-shielded nuclear material detection is possible under Eagle-like conditions; yet, the delayed neutron signal-to-noise ratio, using the current detector designs, was not sufficient to support the real-time detection during an image scan. Of the shield materials investigated using the nominal 6-MeV operation, water was the most challenging due to its photon scattering and neutron moderation/capture issues; however, this shield material does provide the easiest "suspect object" alarm scenario based on the Eagle-developed, correlated cargo image. Additionally, this demonstration showed (as expected) that lead- and water-shielded nuclear material detection, using the delayed neutron signal, is easier with higher beam energies since a larger fraction of the bremsstrahlung spectrum has energy greater than the photofission thresholds of nuclear materials.

In addition to showing nuclear material detection, this technology demonstration identified the challenges involving electron-beam energy operations at or near the photofission threshold energies ( $\sim 6$ $\mathrm{MeV}$ ) of most nuclear materials. These demonstration results were further complicated by higher than expected background levels leading to a reduced signal-to-noise ratio. For example, the background at the demonstration site ( 2134 meters) was a factor of a least 5 higher than that measured at sea level.

Based on the results of this demonstration, this development program has reached a critical design point. Experiments have been sufficiently successful to provide a road map for completing the development of the technology to complement the ARACOR Eagle inspection system with an ability to actively detect shielded and unshielded nuclear materials. More development work needs to be done to move the photonuclear technology to its next logical stage where it will provide a critical new detection capability. The items presented below are key in the successful development of this next stage PPAT capability. 
1. A "lessons-learned" approach with the detection system design is required to enhance the signal-tonoise ratio of the induced signal. This approach would maximize the original design objectives by optimizing the detector system design for:
a. Weight and cost,
b. Reduced natural background contributions,
c. Optimized summation from multiple detector signals, and
d. Maximized prompt and delayed neutron detection.

2. A formal integration plan must be established with ARACOR to effectively implement the PPAT detection system with the Eagle design to develop a practical inspection tool. Such a plan must include a detailed evaluation of all the required modifications to the present nominal 6-MeV Eagle design, a viability assessment of using an operational mode with increased repetition rates, and an assessment of the modifications/issues necessary in the current and future designs to obtain higher electron beam energies. ARACOR is already confident that a higher energy $(>7 \mathrm{MeV})$ version of the Eagle can be designed and is being pursued.

3. Additional experimental efforts must include a natural background characterization at a desired port-of-call and a follow-on demonstration with the optimized detection system.

4. Efforts should be pursued to optimize the prompt neutron emission detection which is at least two orders of magnitude greater than delayed emissions. This detection capability can significantly extend the detection sensitivities at all beam energy operations and should support nuclear material detection simultaneously with the image-acquisition stage of an Eagle inspection. For the prompt neutron detection response to be effective, however, it must effectively differentiate the nuclear material prompt response from the structural material prompt response. An optimized prompt neutron detection approach is considered viable for the ARACOR Eagle design because of its already low dose per accelerator pulse operation and the assumed detection sensitivity per pulse to variations in prompt emissions.

5. Finally, to help identify significant neutron moderation within a container and increase the sensitivity of the detection system, an assessment should be pursued to correlate both induced neutron and gamma-ray signals with the scanned image. This would initially involve an assessment of the induced gamma-ray signal response using an already developed high-purity germanium detection system for use in mixed radiation field environments. This approach would match very well with a parallel effort already being pursued at ARACOR for contraband detection. 


\section{REFERENCES}

1. J. L. Jones, et al., "ARACOR Eagle-matched Operations and Neutron Detector Performance Tests, Formal Report,” INEEL/EXT-02-00823, June 2002.

2. J. L. Jones, et al., "Pulsed Photoneutron Interrogation: The GNT Demonstration System," Formal Report INEL/WINCO-1225, October 1994.

3. J. L. Jones and W. Y. Yoon, Nuclear Smuggling Detection in Cargo Containers: Feasibility Assessment, Formal Report INEL-96/0287, August 1996.

4. J. L. Jones, et al., "Proof-of-Concept Assessment of a Photofission-Based Interrogation System for the Detection of Shielded Nuclear Material," Formal Report INEEL-1523, November 2000.

5. G. J. Arnone, G. S. Brunson, and K. L. Coop, "A Pulse Arrival-Time Recording Module For Analyzing Neutron Multiplicities," Los Alamos National Laboratory report LA-UR-92-3460, presented at the IEEE Nuclear Science Symposium, October 25-31, 1992, Orlando, Florida.

6. R. P. Feynman, F. DeHoffmann, and R. Serber, J. Nucl. Energy 3, 64-69 (1956).

7. A. A. Robba, E. J. Dowdy, and H. F. Atwater, Nucl. Instrum. Methods 215, 473-479 (1983). 